\title{
Nutriquid-Gest: cuestionario estructurado y autoadministrado para evaluar la ingesta alimentaria en mujeres embarazadas. Validación de una encuesta alimentaria
}

Silvia M. García ${ }^{1,2^{*}}$, Silvia Lapertosa ${ }^{3}$, Enzo Rucci ${ }^{1,4}$, Valeria Arias $^{3}$, María V. Fasano $^{5}$ y Peter Kronsbein ${ }^{6}$

${ }^{1}$ CENEXA, Centro de Endocrinología Experimental y Aplicada (UNLP-CONICET), Centro Asociado CICPBA, Facultad de Ciencias Médicas UNLP, La Plata, Argentina; . 'Licenciatura en Nutrición, EURHES, Facultad de Ciencias Médicas, UNLP, La Plata, Argentina; ${ }^{3}$ Facultad de Medicina, UNNE, Corrientes, Argentina; ${ }^{4}$ III-LIDI-Instituto de Investigación en Informática LIDI, Facultad de Informática UNLP, La Plata, Argentina; ${ }^{5}$ Departamento de Farmacología (Cátedra II), Facultad de Medicina, Universidad de Buenos Aires, Buenos Aires, Argentina; ${ }^{6}$ Facultad de Nutrición, Alimentos y Ciencias de Hospitalidad, Niederrhein University of Applied Sciences, Mönchengladbach, Germany

\section{RESUMEN}

Introducción: En Argentina no disponemos de un cuestionario estructurado autoadministrado para evaluar el consumo diario de energía y nutrientes en embarazadas. Objetivo: Diseñar y validar un registro estructurado y autoadministrado adaptado al patrón alimentario de la población argentina para medir ingesta individual de energía y nutrientes en embarazadas (NutriQuid-Gest, [NQ-G]). Materiales y métodos: Diseñamos una encuesta alimentaria estructurada, autoadministrada y un programa de carga de registros para cuantificar automáticamente la ingesta de energía y nutrientes. La validación incluyó: 1) comparación de registros NQ-G completados por voluntarias embarazadas (20 a 40 años) vs. una nutricionista (referencia), y 2) verificación
ABSTRACT

Introduction: In Argentina there is no a structured self-administered questionnaire to evaluate daily energy and nutrient consumption in pregnant women. Objective: To design and validate a structured and self-administered registry adapted to the dietary pattern of the Argentine population, to measure individual energy and nutrient intakes in pregnant women (NutriQuid-Gest, [NQ-G]). Materials and methods: We designed a structured, self-administered food survey and a record-loading program to quantify automatically energy and nutrient intakes. The validation included: 1) comparison of NQ-G records completed by pregnant volunteers (20 to 40 years) vs. a nutritionist (reference), and 2) verification of consumption sensitivity on different days 
de sensibilidad del consumo en diferentes días de la semana. El análisis estadístico incluyó estadística descriptiva, Analysis of variance (ANOVA), coeficiente de correlación intraclase, regresión no paramétrica y clasificación cruzada en quintiles. Resultados: La primera validación (embarazadas voluntarias vs. referencia) mostró una subestimación de $\leq 10 \%$ de la ingesta de energía y de macronutrientes. En la segunda validación 120 embarazadas voluntarias ( $92 \%$ de respuesta) completaron el NQ-G durante siete días consecutivos. La ingesta calórica fue 2,943 $\pm 2,015 \mathrm{kcal} /$ día (media \pm desviación estándar), con un $16 \%$ de proteínas, un $48 \%$ de carbohidratos, un $36 \%$ de lípidos y un $11 \%$ de azúcares refinados. Conclusión: El NQ-G es una herramienta válida, simple, bien aceptada y de bajo costo para evaluar la ingesta alimentaria en mujeres embarazadas.

Palabras clave: Registro estructurado de consumo diario de alimentos. Validación. Evaluación de ingesta en embarazadas. Registro autoadministrado de alimentos. of the week. Statistical analysis: It included descriptive statistics, Analysis of variance (ANOVA), intraclass correlation coefficient, nonparametric regression and cross-classification in quintiles. Results: The first validation (pregnant volunteers vs. reference) showed an underestimation of $\leq 10 \%$ of energy and macronutrient intakes. In the second validation 120 voluntary pregnant women (92\% response) completed the NQ-G for seven consecutive days. The caloric intake was 2,943 $\pm 2,015 \mathrm{kcal} /$ day (mean \pm standard deviation), with $16 \%$ protein, $48 \%$ carbohydrates, $36 \%$ lipids and $11 \%$ refined sugars. Conclusion: NQ-G is a valid, simple, well-accepted and low-cost tool to assess dietary intake in pregnant women. (Rev ALAD. 2019;9:31-43)

Corresponding author: Silvia M. García, silviamgarcia@med.unlp.edu.ar

Key words: Closed-ended food record. Validation. Nutritional assessment in pregnant women. Selfadministered food record.

\section{INTRODUCCIÓN}

La diabetes gestacional (DG), definida como la disglucemia reconocida por primera vez en el embarazo, puede inducir efectos negativos serios tanto en la madre como en el feto. Los descendientes de madres con DG tienen un riesgo mayor de complicaciones al nacer tales como mayor peso, macrosomía y adiposidad, distocia de hombros, hipoglucemia neonatal y obesidad, así como también de desarrollar síndrome metabólico, diabetes tipo 2 (DT2) y enfermedad cardiovascular en la vida adulta ${ }^{1,2}$. En una perspectiva de 5 a 10 años, se estima que el 50\% de las mujeres diagnosticadas con DG desarrollarán DT2 23,4.

La prevalencia de DG está aumentando en todo el mundo en paralelo con la de DT2 y obesidad materna5,6. Este crecimiento está asociado al aumento del número de mujeres con sobrepeso y obesidad que quedan embarazadas ${ }^{7}$. Respecto a esto último, la proporción de mujeres adultas con un IMC $>25 \mathrm{~kg} / \mathrm{m}^{2}$ aumentó a nivel mundial entre 1980 y 2013 del 29.8 al $38.0 \%^{8}$.

Por otra parte, un metaanálisis de 18 ensayos clínicos controlados mostró que la modificación del plan de alimentación de mujeres con DG resultaba en una reducción de la glucemia posprandial y una menor necesidad de medicación para lograrla. Igualmente 
mostró que la modificación del plan de alimentación se asociaba con una reducción sustancial del peso al nacer y un 50\% de reducción del riesgo de macrosomía 9 . Conclusiones similares fueron obtenidas en un metaanálisis recientemente publicado, que mostraba que las intervenciones dietéticas modificaron favorablemente los valores de glucemia y peso al nacer ${ }^{10}$.

En función de lo expuesto resulta evidente que la evaluación nutricional de la mujer al inicio y a lo largo del embarazo debería ser una práctica sistemática en la consulta obstétrica para asegurar que se cumplan las recomendaciones de la Dirección Nacional de Maternidad e Infancia y lograr así óptimas condiciones para su curso y terminación ${ }^{11}$.

En este contexto, verificar la ingesta alimentaria es un recurso eficaz para identificar tempranamente hábitos no saludables, así como también para evaluar resultados de una intervención educativa que promueva la adopción de hábitos nutricionales saludables ${ }^{12}$.

Para la evaluación alimentaria de cohortes se requiere un instrumento que refleje hábitos alimentarios culturales locales, estime cuantitativamente el consumo de energía y nutrientes específicos y facilite el proceso de registro, minimizando la carga de datos y economizando recursos humanos y económicos. En este sentido existen diversos instrumentos de evaluación de la ingesta, tales como cuestionarios de frecuencia de consumo (CFC), recordatorios de 24 horas, registro de consumo diario, historia dietética, encuestas breves y combinaciones de estos. Todos ellos tienen fortalezas y debilidades que afectan a su aplicación específica ${ }^{13,14}$.

Los CFC se usan frecuentemente en estudios epidemiológicos con lapsos de evaluación retrospectiva variables, pero su uso se considera inapropiado para estimar parámetros cuantitativos actuales a nivel individual' ${ }^{13,14}$.

El recordatorio de 24 horas permite obtener resultados cuantitativos, pero requiere un entrenamiento de los entrevistadores y varios días de entrevista dentro de un lapso de varios meses para determinar la ingesta habitual; implica además una alta carga de trabajo en codificación y procesamiento de datos.

El registro del consumo de alimentos no estructurado, también conocido como registro diario de alimentos, provee resultados cuantitativos de la ingesta de alimentos y nutrientes, pero para obtener una evaluación representativa de un periodo de tiempo, requiere registrar tres días de la semana o siete días consecutivos $^{14}$. Su fortaleza es que proporciona información cuantitativa, pero el participante debe estar motivado para registrar adecuada y detalladamente el total de alimentos y bebidas consumidas y el equipo evaluador debe interpretar y procesar los datos, lo que implica una alta carga de trabajo. El número de registros completos y la validación de la información disminuye cuando el registro se extiende más de cuatro días ${ }^{13,15}$. Además, los registros deben ser interpretados por un o una nutricionista antes de ingresar la información a la base de datos $^{16}$. Estos aspectos limitan su uso en proyectos que incluyen grandes cohortes.

Las encuestas estructuradas de registro de consumo de alimentos autoadministradas facilitan la captura de datos, eliminan el trabajo de interpretación y simplifican la carga de datos, además el empleo de listas de alimentos y porciones automatizan la obtención de resultados. Por estas características constituyen una herramienta apta para estudios de grupos poblacionales. Lamentablemente, no hemos encontrado en Argentina una encuesta autoadministrada validada para evaluar la ingesta diaria en embarazadas; lo más aproximado hallado fue un 
registro estructurado, autoadministrado y validado para cuantificar la ingesta diaria de energía y nutrientes en adultos de 45 a 75 años (NutriQuid), diseñado por nuestro grupo para utilizarse en un programa de prevención primaria de diabetes ${ }^{17,18}$ (su formulario está disponible en http://www.ppdba. cenexa.org/nutriquid).

En función de lo expuesto, el objetivo del presente estudio fue desarrollar (con base en el modelo NutriQuid) un modelo de registro estructurado y autoadministrado para cuantificar el consumo diario de energía y nutrientes en mujeres embarazadas (NutriQuid-Gest, [NQ-G]) y validar su implementación en nuestro medio.

\section{MATERIALES Y MÉTODOS}

\section{Diseño de la encuesta}

Un equipo interdisciplinario (nutricionistas, médicos e informáticos) diseñó un modelo de encuesta estructurada para registro de consumo de alimentos en mujeres embarazadas (NQ-G). El modelo contiene 62 renglones que incluyen alimentos representativos de nuestro ámbito cultural alimentario. Seleccionamos, agrupamos e incluimos dichos alimentos considerando las características de su consumo habitual en Argentina, el régimen diario de comidas y las características nutricionales de nuestra población adulta ${ }^{19-22}$.

En algunos casos de comidas preparadas (p. ej., pizza, tarta y empanadas), el equipo de nutricionistas calculó el contenido de energía y nutrientes de porciones estandarizadas, usando la información de la base de datos de CENEXA ${ }^{26}$ y Argenfood ${ }^{27}$. El contenido de azúcares libres fue calculado utilizando la información declarada por los fabricantes.
Estas condiciones permiten cuantificar los macro y micronutrientes claves en el embarazo, como hidratos de carbono, proteínas, grasas, fibra, minerales y vitaminas. En este sentido, en comparación a su predecesor Nutri-Quid, el NQ-G presenta mayores especificaciones, tales como: a) incorpora pan integral y con salvado para contabilizar fibra, vitaminas y minerales; b) discrimina vísceras (hígado, corazón y riñón), que constituyen una importante fuente de hierro y vitaminas, y c) incluye ítems para determinar la ingesta de suplementos de hierro y ácido fólico, cuyo consumo en embarazadas está indicado por el Ministerio de Salud de la Nación ${ }^{11}$. También incluye tres preguntas para estimar el consumo de sal como condimento y de aceite como aderezo. La tabla 1 muestra una sección del NQ-G, mientras que su versión completa puede ser descargada de http:// www.qualidiab-gest.cenexa.org/nutriquidgest.

El registro NQ-G consiste en cuatro columnas verticales:

- La primera columna de la izquierda incluye la lista de alimentos descritos en el párrafo anterior.

- La segunda columna permite seleccionar con un círculo alguna característica diferencial del alimento (p. ej., pan común/integral/sin sal).

- La tercera columna define el tamaño de la porción de cada ítem. La porción de carne, pasta, cereales y sal está acompañada con un dibujo. Para los demás ítems se utilizaron medidas caseras como cucharaditas, cucharadas, tazas y unidades comunes.

- La cuarta columna registra el total de porciones consumidas diariamente.

Al final de cada página hay un recordatorio para revisar lo registrado en la segunda y en la cuarta 
TABLA 1. Sección representativa del NutriQuid-Gest

\begin{tabular}{|l|l|l|l|}
\hline Alimentos & $\begin{array}{l}\text { Tipo } \\
\text { (rodear con un círculo) }\end{array}$ & Una porción es igual a & Porciones/día (0, 1/2, 1, 2...) \\
\hline Pan & Común/Integral/Sin sal & 1 mignon (50 g) & \\
\hline Pan de molde & Lactal/Con salvado/Sin sal & 1 rodaja tipo lactal & \\
\hline Bizcochito de grasa, tortas fritas & & 1 unidad & \\
\hline Chipá & & 1 unidad & \\
\hline Galletitas dulces & Simples/Rellenas & 1 unidad & 1 unidad \\
\hline Otras galletitas, galletas de arroz & Comunes/Sin sal & & \\
\hline
\end{tabular}

columna que sirve para controlar y evitar posibles omisiones.

La información registrada en el NQ-G por las personas encuestadas se incorporó en la base de datos MySQL23 usando un software específico desarrollado mediante el Symfony 1.4 framework ${ }^{24}$.

Los datos incorporados en la base de datos se analizaron posteriormente empleando el paquete de software de SARA (Sistema de Análisis y Registro de Alimentos, Ministerio de Salud de la Nación)25, que calcula la ingesta de nutrientes en función de la cantidad total de porciones de cada ítem registrado. El sistema desarrollado cuantifica el consumo total de energía, proteínas, carbohidratos, lípidos, ácidos grasos poliinsaturados, monoinsaturados y saturados, colesterol, fibra dietética, sodio, potasio, calcio, fósforo, hierro, zinc, azúcares libres, porciones de frutas y verduras y las vitaminas A, B1, B2, niacina, ácido fólico, B12 y C.

\section{Validación de la encuesta de registro alimentario NQ-G}

Este proceso se desarrolló en dos fases.

\section{Primera fase. Verificación DE LA PRECISIÓN DE LA ENCUESTA}

Siguiendo el modelo utilizado previamente para validar el NutriQuid ${ }^{17}$ se compararon los registros NQ-G completados por las participantes del estudio con los completados simultáneamente por una nutricionista (referencia), que había diseñado las comidas y conocía la composición nutricional de los alimentos ofrecidos a las participantes del estudio.

Esta etapa se desarrolló entre febrero y mayo del 2017, en la sala de alto riesgo del Hospital Materno Neonatal Eloísa Torrent de Vidal de la ciudad de Corrientes, Provincia de Corrientes (Argentina), e incluyó 50 gestantes voluntarias de entre $20 \mathrm{y}$ 40 años internadas, sin enfermedades metabólicas crónicas, desórdenes nutricionales, cáncer ni cirugías recientes, que recibían una dieta estándar.

En la mañana de la prueba de validación, las participantes recibieron el formulario NQ-G con su instructivo y se les solicitó registrar todas las comidas y bebidas provistas durante el día por el servicio de alimentación del hospital. Simultáneamente, la nutricionista realizó la misma tarea, registrando en el 
formulario NQ-G los alimentos que fueron servidos a cada participante a lo largo del día (referencia).

Aunque el registro fue anónimo, se solicitó a las participantes consignar los cuatro últimos números de su documento nacional de identidad para evitar superposición de datos de diferentes personas y facilitar su análisis posterior.

\section{SEgunda faSE: VERIFICACIÓN DE LA SENSIBILIDAD PARA IDENTIFICAR POSIBLES DIFERENCIAS EN EL CONSUMO DE ALIMENTOS EN DIFERENTES DÍAS DE LA SEMANA}

La segunda fase fue completada por las participantes durante siete días utilizando el NQ-G.

El tamaño de la muestra se determinó buscando un $5 \%$ de diferencia en la ingesta de energía total y asumiendo un 5\% de error alfa y un $20 \%$ de error beta, lo que resultó en 106 participantes. Considerando la posibilidad de entrega de encuestas incompletas o abandonos, este número se incrementó a 130 participantes.

Esta fase se llevó a cabo entre los meses de febrero y mayo de 2017 e incluyó 120 mujeres embarazadas voluntarias de 20 a 40 años de edad, con atención sanitaria ambulatoria (consultorio externo). Las participantes fueron reclutadas en el Hospital Materno Neonatal Eloísa Torrent de Vidal, la Maternidad del Hospital Ángela Iglesia de Llano y en los Centros de Atención Primaria de la Salud (CAP) N..$^{\circ}$ VIII B ${ }^{\circ}$ Santa Teresita, CAP N. ${ }^{\circ} \mathrm{X} \mathrm{B}^{\circ}$ Molina Punta, instituciones pertenecientes al Ministerio de Salud de la provincia de Corrientes (Argentina).

Las participantes recibieron siete formularios NQ-G (uno para cada día de la semana) y su correspon- diente instructivo, solicitándoles registrar diariamente el consumo de alimentos durante una semana. Para evitar el sesgo de fatiga, el día de inicio de registro fue variando.

Como en la fase 1 , los registros se completaron de manera anónima, pero se solicitó anotar los cuatro últimos números del documento nacional de identidad en la planilla de NQ-G para evitar superposición de datos y facilitar el análisis posterior.

\section{ANÁLISIS ESTADÍSTICO}

- Primera fase: se usaron técnicas de estadística descriptiva para estimar el consumo diario de energía, carbohidratos, proteínas, grasa y micronutrientes a partir de los registros en el NQ-G. Subsecuentemente, se calculó el porcentaje de diferencia entre los valores obtenidos por los participantes voluntarios y la referencia. También se realizó validación cruzada por quintiles para energía, carbohidratos, proteínas y grasa. Finalmente se calculó el coeficiente de correlación de Pearson para evaluar la relación entre los registros de los participantes y los de la referencia.

- Segunda fase (7 días consecutivos de registro individual): para evaluar diferencias entre registros diarios individuales se utilizó el modelo ANOVA para medidas repetidas; también el coeficiente de correlación intraclase $(\mathrm{CCl})$ a partir de un modelo ANOVA mixto de dos vías como medida de la fiabilidad test-retest de cada variable (energía total y diferentes nutrientes). El CCI evaluó la reproducibilidad de las mediciones diarias de la misma persona para cada componente evaluado (energía total y varios nutrientes) a lo largo de la semana de estudio. 
Las variables cuantitativas se expresaron como media \pm desvío estándar (DE) o error estándar (EE) (en cada caso se especificó cuál de ellos se utilizó). Las diferencias encontradas se consideraron significativas cuando su valor de $p$ era $\leq 0.05$.

\section{DECLARACIÓN DE ÉTICA}

Este estudio se realizó siguiendo las directrices de la Declaración de Helsinki y todos los procedimientos con seres humanos fueron aprobados por el Comité de Ética de la Facultad de Medicina de la Universidad Nacional de Nordeste (UNNE).

\section{RESULTADOS}

\section{Primera fase}

Participaron voluntariamente y completaron el NQ-G durante 24 h de internación 50 mujeres embarazadas de 20 a 40 años. Siete de estos registros (14\%) fueron excluidos del análisis por no haber cumplido estrictamente el instructivo provisto y haber incurrido en diversos errores tales como: a) no recibir la bandeja/comida apropiada; b) por falta de apetito no comer toda la comida provista y solamente anotar lo ingerido, y c) anotar comidas adicionales que recibieron fuera de las provistas por el servicio de alimentación del hospital. En consecuencia, solo se utilizaron 43 de los cuestionarios que estaban completos para el análisis estadístico.

Una nutricionista entrenada (referencia) completó simultáneamente su registro NQ-G y los registros fueron luego comparados. Dicha comparación mostró una diferencia no superior al 10\% en el registro de la ingesta de energía, cantidad de proteínas, hidratos de carbono y otros nutrientes (Tabla 2).
El registro de lípidos en cambio mostró una subestimación del $16 \%$.

Los coeficientes de correlación de los valores relativos [\%E] de macronutrientes entre los participantes y la referencia variaron 0.61 para los carbohidratos, 0.67 para las proteínas y 0.69 para los lípidos (Tabla 2).

La clasificación cruzada de ingesta total de energía, carbohidratos, proteínas y grasas (g/día y \% E) determinada por el NQ-G completado por los participantes vs. la referencia se muestran en la Tabla 3. La proporción de personas cuyas respuestas se ubicaron exactamente en el mismo quintil que la referencia oscilaron entre el 30\% (carbohidratos) y el $61 \%$ (proteínas). Según esta metodología, un promedio menor al 2\% clasificó erróneamente en los quintiles extremos (Tabla 3).

\section{Segunda fase}

De las 130 mujeres embarazadas invitadas a participar, 120 (92\%) devolvieron completos los registros, obteniéndose un total de 840 encuestas correspondientes al registro diario de ingesta de alimentos de siete días consecutivos (Tabla 4).

Mientras el tiempo requerido por un operador para cargar cada registro en la base de datos fue variable (entre 2 y 6 minutos), la obtención de resultados fue inmediata: el programa convirtió automáticamente los datos cargados en valores de consumo de energía.

Los datos registrados mostraron que la ingesta calórica diaria promedio fue de 2,943 $\pm 184 \mathrm{kcal} /$ día (media $\pm \mathrm{EE}$ ). De ese total de energía las proteínas representaron el $16 \%$, los carbohidratos el $48 \%$ y los lípidos el 36\%. Los azúcares refinados constituyeron 
TABLA 2. Comparación de valores de nutrientes empleando el NutriQuid-Gest, completado por nutricionistas (referencia) vs. mujeres embarazadas (participantes) (primera fase de validación)

\begin{tabular}{|c|c|c|c|c|c|c|c|c|}
\hline \multirow[t]{2}{*}{ Variable } & \multicolumn{2}{|c|}{ Referencia } & \multicolumn{2}{|c|}{ Participante } & \multirow[b]{2}{*}{ valor de $p$} & \multicolumn{2}{|c|}{ Diferencia ref. vs. part. (\%) } & \multirow{2}{*}{$\begin{array}{l}\text { Coeficiente } \\
\text { de Pearson }\end{array}$} \\
\hline & Media & DE & Media & DE & & Media & DE & \\
\hline Energía (kcal/d) & 2,305 & 223 & 2,156 & 207 & $<0.0001$ & -6.2 & 7.6 & 0.65 \\
\hline Carbohidratos (g/d) & 286 & 41 & 273 & 43 & 0.027 & -3.7 & 13.6 & 0.62 \\
\hline Carbohidratos (\%E) & 49.5 & 3.9 & 50.7 & 6.0 & 0.110 & 2.4 & 9.6 & 0.61 \\
\hline Proteínas (g/d) & 110 & 11 & 113 & 15 & 0.040 & 3.0 & 9.1 & 0.74 \\
\hline Proteínas (\%E) & 19.1 & 1.4 & 21.0 & 2.4 & $<0.0001$ & 10.1 & 9.5 & 0.67 \\
\hline Lípidos (g/d) & 80 & 12 & 68 & 17 & $<0.0001$ & -15.8 & 16.6 & 0.67 \\
\hline Lípidos (\%E) & 31.4 & 3.8 & 28.3 & 6.5 & 0.0001 & -10.2 & 16.1 & 0.69 \\
\hline AG saturados (g/d) & 20.6 & 3.5 & 23.8 & $5 \cdot 9$ & $<0.0001$ & 15.5 & 21.7 & 0.68 \\
\hline AG monoinsat. (g/d) & 24.9 & 3.5 & 23.6 & 5.9 & 0.056 & -5.5 & 19.1 & 0.58 \\
\hline AG poliinsat. (g/d) & 28.5 & 6.6 & 14.2 & $7 \cdot 3$ & $<0.0001$ & -51.3 & 23.3 & 0.66 \\
\hline Colesterol (mg/d) & 321 & 60 & 387 & 105 & $<0.0001$ & 21.9 & 30.6 & 0.47 \\
\hline Fibra (g/d) & 19.6 & 5.0 & 21.2 & 6.6 & 0.072 & 11.4 & 31.7 & 0.52 \\
\hline Sodio (mg/d) & 3,737 & 1,086 & 1,997 & 1,131 & $<0.0001$ & -37.5 & 49.7 & 0.25 \\
\hline Potasio $(\mathrm{mg} / \mathrm{d})$ & 3,758 & 570 & 3,913 & 891 & 0.160 & 4.5 & 17.8 & 0.61 \\
\hline Calcio (mg/d) & 980 & 137 & 928 & 191 & 0.497 & -5.0 & 16.3 & 0.52 \\
\hline Fósforo (mg/d) & 1,676 & 156 & 1,763 & 225 & 0.909 & -0.1 & 10.0 & 0.67 \\
\hline Hierro (mg/d) & 17.3 & 5.1 & 18.1 & 4.4 & 0.387 & 8.1 & 24.7 & 0.30 \\
\hline Zinc (mg/d) & 17.7 & 2.2 & 18.3 & $3 \cdot 3$ & 0.206 & 4.2 & 17.5 & 0.41 \\
\hline Vitamina A ( $\mu \mathrm{g} / \mathrm{d})$ & 795 & 221 & 688 & 209 & 0.005 & -10.0 & 26.3 & 0.39 \\
\hline Vitamina B1 (mg/d) & 2.6 & 0.7 & 2.5 & 0.8 & 0.242 & -2.2 & 19.8 & 0.79 \\
\hline Vitamina B2 (mg/d) & 3.1 & 0.7 & 3.1 & 0.8 & 0.823 & 1.1 & 23.0 & 0.70 \\
\hline Niacina $(\mathrm{mg} / \mathrm{d})$ & 33.3 & 6.7 & 32.7 & 7.1 & 0.279 & -1.5 & 12.7 & 0.83 \\
\hline Folatos $(\mu \mathrm{g} / \mathrm{d})$ & 472 & 123 & 496 & 140 & 0.303 & 8.2 & 30.0 & 0.38 \\
\hline Vitamina B12 ( $\mu \mathrm{g} / \mathrm{d})$ & 6.6 & 1.4 & 7.4 & 1.6 & 0.0002 & 15.5 & 31.1 & 0.63 \\
\hline Vitamina C (mg/d) & 367 & 323 & 410 & 310 & 0.364 & 53.0 & 109 & 0.55 \\
\hline $\begin{array}{l}\text { Frutas y verduras } \\
\text { (porciones/d) }\end{array}$ & 3.4 & 1.8 & 3.5 & 1.8 & 0.620 & 15.2 & 47.6 & 0.56 \\
\hline
\end{tabular}

el $11 \%$ de la ingesta calórica total. También puede observarse que el día domingo corresponde al de mayor ingesta de energía y nutrientes. El fenómeno se visualiza más claramente en la figura 1. Los CCI se mantuvieron en un rango entre 0.52 y 0.78 .

\section{DISCUSIÓN}

Los resultados presentados demuestran la utilidad del NQ-G para evaluar en gran escala la ingesta alimentaria de mujeres embarazadas. 
TABLA 3. Clasificación cruzada de ingesta de nutrientes determinada por el NQ-G completado por las embarazadas (participantes) vs. por las nutricionistas (referencia)

\begin{tabular}{|l|c|c|c|}
\hline Nutriente & Quintil coincidente & Quintil coincidente y adyacente & Quintil opuesto \\
\hline Energía & $54 \%$ & $81 \%$ & $0 \%$ \\
\hline Carbohidratos & $30 \%$ & $77 \%$ & $2 \%$ \\
\hline Carbohidratos (\%E) & $33 \%$ & $77 \%$ & $5 \%$ \\
\hline Proteínas & $61 \%$ & $80 \%$ & $0 \%$ \\
\hline Proteínas (\%E) & $44 \%$ & $86 \%$ & $2 \%$ \\
\hline Lípidos & $56 \%$ & $84 \%$ & $2 \%$ \\
\hline Lípidos (\%E) & $49 \%$ & $77 \%$ & $0 \%$ \\
\hline
\end{tabular}

TABLA 4. Consumo diario de energía y nutrientes promedio durante una semana consecutiva utilizando el registro estructurado de consumo de alimentos NutriQuid-Gest

\begin{tabular}{|c|c|c|c|c|c|c|c|c|}
\hline Variables & Lunes & Martes & Miércoles & Jueves & Viernes & Sábado & Domingo & $\begin{array}{c}\mathrm{CCl} \\
(95 \% \mathrm{Cl} \mathrm{CCl})\end{array}$ \\
\hline $\begin{array}{l}\text { Energía } \\
\qquad(\mathrm{kcal} / \mathrm{d} \text { áa)* }\end{array}$ & $3,005 \pm 856$ & $2,756 \pm 818$ & $3,023 \pm 938$ & $2,707 \pm 816$ & $2,999 \pm 912$ & $2,864 \pm 841$ & $3,248 \pm 960$ & $\begin{array}{c}0.75 \\
(0.67-0.81)\end{array}$ \\
\hline $\begin{array}{l}\text { Carbohidratos } \\
\left(\mathrm{g} / \text { día }^{\dagger}\right.\end{array}$ & $381 \pm 111$ & $360 \pm 116$ & $394 \pm 142$ & $358 \pm 110$ & $399 \pm 138$ & $356 \pm 118$ & $408 \pm 137$ & $\begin{array}{c}0.78 \\
(0.72-0.84)\end{array}$ \\
\hline $\begin{array}{l}\text { Proteínas } \\
(\mathrm{g} / \mathrm{día})^{\ddagger}\end{array}$ & $118 \pm 33$ & $111 \pm 31$ & $119 \pm 33$ & $111 \pm 27$ & $119 \pm 32$ & $109 \pm 29$ & $126 \pm 34$ & $\begin{array}{c}0.68 \\
(0.58-0.76)\end{array}$ \\
\hline $\begin{array}{l}\text { Lípidos } \\
\text { (g/día)§ }\end{array}$ & $122 \pm 58$ & $107 \pm 47$ & $118 \pm 51$ & $102 \pm 50$ & $113 \pm 47$ & $118 \pm 50$ & $131 \pm 61$ & $\begin{array}{c}0.52 \\
(0.38-0.64)\end{array}$ \\
\hline $\begin{array}{l}\text { Fibra } \\
\text { (g/día) }\end{array}$ & $25 \pm 9$ & $24 \pm 10$ & $24 \pm 10$ & $23 \pm 9$ & $25 \pm 10$ & $22 \pm 9$ & $25 \pm 9$ & $\begin{array}{c}0.64 \\
(0.54-0.73)\end{array}$ \\
\hline $\begin{array}{l}\text { Sodio } \\
\text { (mg/día) }\end{array}$ & $3,660 \pm 1,633$ & $3,348 \pm 1,484$ & $3,694 \pm 1,756$ & $3,453 \pm 1,400$ & $3,883 \pm 1,824$ & $3,609 \pm 1,619$ & $4,038 \pm 1,669$ & $\begin{array}{c}0.64 \\
(0.53-0.73)\end{array}$ \\
\hline $\begin{array}{l}\text { Potasio } \\
\text { (mg/día) }\end{array}$ & $4,085 \pm 1,333$ & $3,863 \pm 1,330$ & $4,026 \pm 1,378$ & $3,924 \pm 1,208$ & $4,095 \pm 1,386$ & $3,695 \pm 1,101$ & $4,296 \pm 1,309$ & $\begin{array}{c}0.62 \\
(0.51-0.72)\end{array}$ \\
\hline $\begin{array}{l}\text { Azúcar } \\
\text { refinado } \\
\text { (g/día) }\end{array}$ & $81 \pm 61$ & $61 \pm 48$ & $81 \pm 71$ & $65 \pm 51$ & $76 \pm 62$ & $91 \pm 74$ & $90 \pm 67$ & $\begin{array}{c}0.73 \\
(0.65-0.80)\end{array}$ \\
\hline
\end{tabular}

Los valores representan media $\pm \mathrm{DE}$.

${ }^{*} \mathrm{p}<0.001 \Delta$ diferencia significativa lunes vs. jueves; martes vs. domingo, miércoles vs. jueves; jueves vs. viernes; jueves vs. domingo; sábado vs. domingo.

${ }^{\dagger} \mathrm{p}<0.001 \Delta$ diferencia significativa martes vs. domingo; jueves vs. domingo y sábado vs. domingo; los valores indicados de carbohidratos incluyen fibra.

${ }^{\ddagger} \mathrm{p}<0.001 \Delta$ diferencia significativa martes vs. domingo; jueves vs. domingo: sábado vs. domingo.

$\S_{\mathrm{p}}<0.001 \Delta$ diferencia significativa martes vs. domingo; jueves vs. domingo.

१p < $0.001 \Delta$ diferencia significativa lunes vs. martes; martes vs. sábado; martes vs. domingo; jueves vs. sábado; viernes vs. domingo.

$\mathrm{CCl}$ : coeficiente de correlación intraclase $(95 \% \mathrm{Cl}$ for $\mathrm{CCl})$.

La metodología utilizada en la fase 1 de validación somete a la herramienta NQ-G a un gran desafío: comparar el registro completado por un experto en nutrición, que además indicó la preparación de la comida (nutricionista-referencia), con los registros realizados por alguien sin experiencia en este 


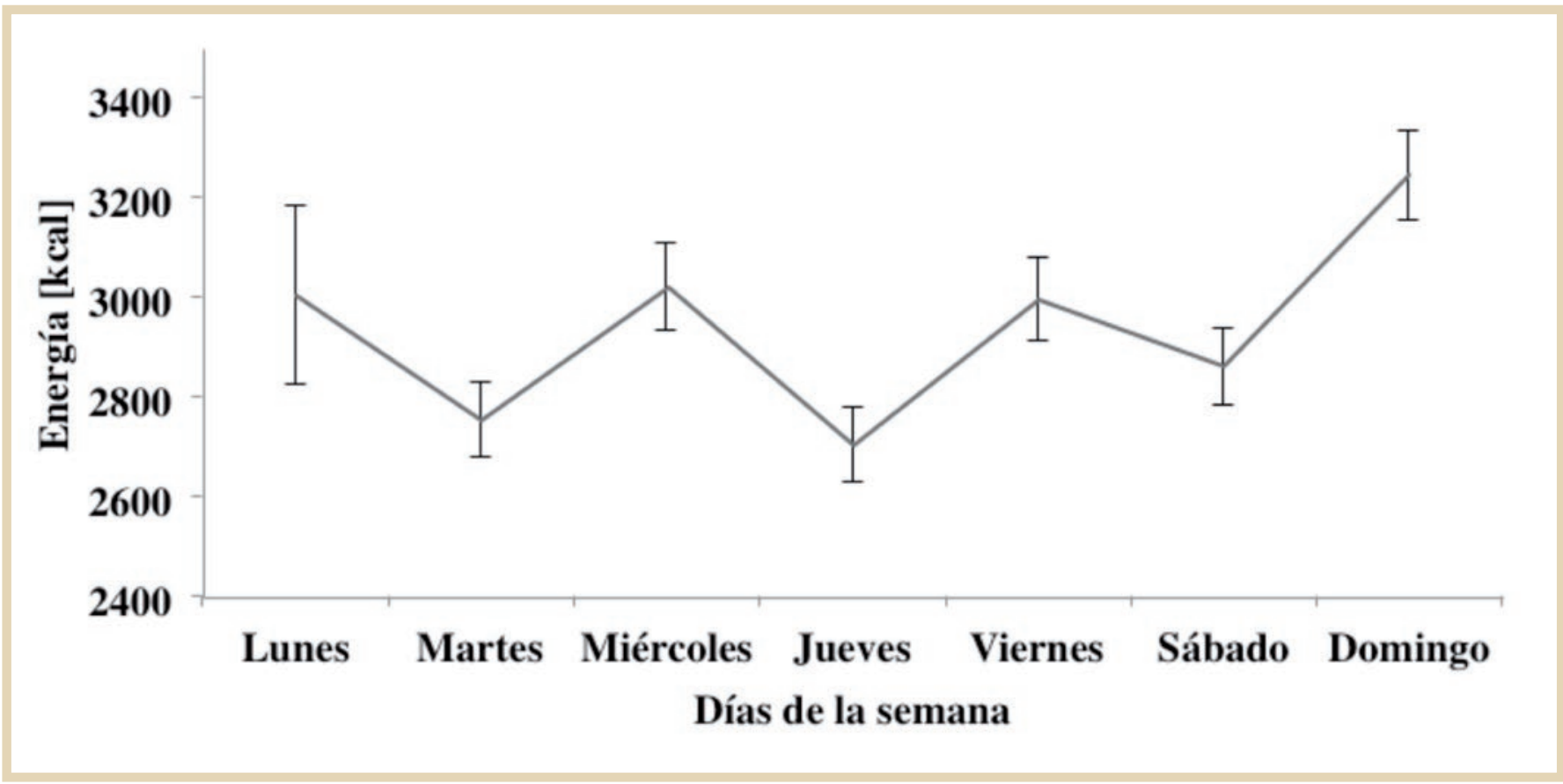

Figura 1. Ingesta calórica a lo largo de la semana (segunda fase de validación).

$\mathrm{n}=120$ casos (mujeres embarazadas) para todos los parámetros. Los valores representan la media $\pm \mathrm{EE}$.

campo (las participantes). Dicha comparación mostró una diferencia inferior al $10 \%$ en la ingesta de energía y la mayor parte de los nutrientes, y una alta correlación entre los valores de macronutrientes ajustados al valor energético total registrados por las participantes y la referencia. Esto sugeriría un alto grado de comprensión de las preguntas del NQ-G por las embarazadas participantes.

Aunque las diferencias entre participantes y referencia fueron mayores en el registro del consumo de lípidos, ácidos grasos poliinsaturados, sodio y azúcar refinado, ello sería debido a que las comidas ofrecidas ya estaban condimentadas y alineadas. Consecuentemente, mientras las nutricionistas que completaron el NQ-G conocían con precisión el agregado de aceite, sal y azúcar, las participantes al ignorarlo no lo registraron.

La subestimación en la ingesta de energía, nutrientes y especialmente sodio, azúcar refinado, ácidos grasos poliinsaturados y lípidos totales ha sido previamente reportada por otros autores empleando metodologías diferentes a la nuestra en adultas no embarazadas ${ }^{28,29}$. Nuestro subregistro, sin embargo, fue inferior al $45 \%$ reportado en embarazadas ${ }^{34}$, por lo que nuestros resultados se aproximan mejor a las recomendaciones establecidas para embarazadas en diferentes países de la región ${ }^{35,36}$.

Para solucionar particularmente la subestimación del aceite vegetal, se suele omitir su inclusión en los listados de alimentos y considerar una porción estandarizada según el patrón de consumo habitual de la población local. Por ejemplo, Rodríguez, et al., en España, excluyeron el aceite en dicho listado basándose en que su consumo en diversos platos y varias veces al día dificultaba su estimación y utilizaron una porción estandarizada de 46 g/día de aceite vegetal en la validación de un cuestionario de frecuencia de consumo ${ }^{30,31}$. 
La aceptabilidad de NQ-G por las participantes fue buena, ya que $92 \%$ de las encuestas semanales fueron completadas adecuadamente en la segunda fase de validación, demostrando el patrón característico de ingesta de calorías y macronutrientes de nuestra población objetivo. Complementariamente, el software desarrollado para determinar la ingesta de nutrientes en el NQ-G permitió su identificación inmediata (Tabla 4).

Mientras la ingesta energética semanal promedio registrada (2,943 kcal/día) estuvo por encima de los valores recomendados ${ }^{32,33}$, la distribución porcentual de macronutrientes mostró valores cercanos a los recomendados, con discreto aumento del consumo de grasas a expensas de proteínas e hidratos de carbono; los azúcares refinados constituyeron el $11 \%$ de la ingesta calórica total.

La sensibilidad del NQ-G permitió identificar pequeños cambios en el consumo diario de energía y nutrientes entre los días de la semana con un mayor consumo en el día domingo. El aumento del consumo de los fines de semana ha sido también descrito en estudios realizados en adultos ${ }^{17,37}$.

En función de estos resultados, el registro NQ-G dos días de la semana y el día domingo brindaría resultados consistentes, lo que reduce simultáneamente la carga de trabajo representada por su empleo durante los siete días de la semana y la fatiga de registro, tal como lo sugieren otros autores ${ }^{13-15}$.

Las encuestas autoadministradas son económicamente ventajosas comparadas con otros métodos, pues ahorran tiempo en entrevistas, reducen el tiempo y el costo de implementación, y su precisión es relativamente suficiente, todos elementos importantes en estudios poblacionales ${ }^{38,39}$. En nuestro caso disminuye también el tiempo de carga de los registros en la base de datos, que fue de 2 a 6 minutos según la habilidad del operador.

Por todas las características descritas, la utilización del NQ-G permitiría cubrir una demanda parcialmente cubierta: la verificación sistemática y en gran escala de la ingesta alimentaria de mujeres embarazadas en el primer nivel de atención y la corrección de falencias detectadas en forma precoz y efectiva. El dato no es menor considerando que dicha ingesta y el estado nutricional de la mujer embarazada impactan esencialmente sobre la salud materna y el desarrollo fetal ${ }^{40,41}$. La malnutrición, frecuente en nuestro medio en población adulta y en este caso en la mujer antes y durante el embarazo, aumenta el riesgo de desarrollar enfermedades tales como la diabetes en la madre y la morbimortalidad fetal y posparto, al igual que en el futuro del recién nacido. Diversos estudios y en particular uno reciente de cohorte observacional y poblacional con más de 1.2 millones de nacidos vivos, destacan el aumento del riesgo de anomalías congénitas debido al sobrepeso y obesidad materna ${ }^{42}$.

A pesar de sus ventajas, el registro NQ-G tiene algunas limitaciones tales como: a) para completarlo las personas deben estar alfabetizadas o contar con asistencia para hacerlo; b) la selección de alimentos incluidos en la encuesta se basó en el consumo habitual de alimentos en Argentina ${ }^{19-22}$, por lo que su empleo fuera de nuestro país debería adaptarse a las condiciones locales en regiones, y c) el número comparativamente bajo de alimentos en el NQ-G (apropiado para nuestra región y para disminuir la carga de las encuestadas) requeriría igual adaptación para su empleo fuera de este ámbito.

En conclusión, el NQ-G es una herramienta fácil de emplear y de bajo costo que permite una evaluación razonablemente precisa de la ingesta alimentaria en población de mujeres embarazadas en Argentina. 
Se necesitan más estudios para demostrar sus beneficios y aplicabilidad en diferentes condiciones patológicas de la embarazada.

\section{AGRADECIMIENTOS}

Los autores agradecen la desinteresada colaboración de la Lic. Mariana Prestes en la evaluación estadística de los datos y del Prof. J.J. Gagliardino por su continuo asesoramiento durante el desarrollo del estudio y preparación del manuscrito.

\section{CONTRIBUCIÓN DE LOS AUTORES}

SMG participó en el diseño de la encuesta, en la planificación de las actividades de validación y en la preparación del manuscrito; SL y VA monitorearon la recolección de datos y actividades de la nutricionista de referencia y la carga de datos en la base de datos; ER desarrolló el software del NQ-G y administró la base de datos; MVF tuvo a su cargo la supervisión del análisis estadístico del estudio y PK participó en el diseño de la encuesta, el análisis de datos y en la preparación del manuscrito.

\section{BIBLIOGRAFÍA}

1. Catalano PM. The impact of gestational diabetes and maternal obesity on the mother and her offspring. J Developmental Origins of Health and Disease. 2010;1:208-15.

2. Gillman MW, Rifas-Shiman S, Berkey CS, Field AE, Colditz GA. Maternal gestational diabetes, birth weight, and adolescent obesity. Pediatrics. 2003;111:e221-6.

3. Kim C, Newton KM, Knopp RH. Gestational diabetes and the incidence of type 2 diabetes: a Systematic Review. Diabetes Care. 2002; 25:1862-8.

4. Damm P, Houshmand-Oeregaard A, Kelstrup L, Lauenborg J, Mathiesen ER, Clausen TD. Gestational diabetes mellitus and longterm consequences for mother and offspring: a view from Denmark. Diabetologia. 2016;59:1396-9.

5. Bottalico JN. Recurrent gestational diabetes: risk factors, diagnosis, management, and implications. Semin Perinatol. 2007;31:176-84.

6. Dabelea D, Snell-Bergeon JK, Hartsfield CL, Bischoff KJ, Hamman RF, McDuffie RS; Kaiser Permanente of Colorado GDM Screening Program. Increasing prevalence of estational diabetes mellitus (GDM) over time and by birth cohort: Kaiser Permanente of Colorado GDM Screening Program. Diabetes Care. 2005;28:579-84.
7. Kim SY, England L, Wilson HG, Bish C, Satten GA, Dietz P. Percentage of gestational diabetes mellitus attributable to over-weight and obesity. Am J Public Health. 2010;100:1047-52.

8. Ng M, Fleming T, Robinson M, Thomson B, Graetz N, Margono C, et al. Global, regional, and national prevalence of overweight and obesity in children and adults during 1980-2013: a systematic analysis for the Global Burden of Disease Study 2013. Lancet. 2014;384:766-81.

9. Hernandez TL, Brand-Miller JC. Nutrition therapy in gestational diabetes mellitus: Time to move forward. Diabetes Care. 2018;41:1343-5.

10. Yamamoto JM, Kellett JE, Balsells M, García-Patterson A, Hadar E, Solà I, et al. Gestational diabetes mellitus and diet: A systematic review and meta-analysis of randomized controlled trials examining the impact of modified dietary interventions on maternal glucose control and neonatal birth weight. Diabetes Care. 2018;41:1346-61.

11. Ministerio de Salud de la Nación. Nutrición y Embarazo. Recomendaciones en Nutrición para los equipos de salud. Dirección Nacional de Maternidad e Infancia. Buenos Aires: Ministerio de Salud; 2012.

12. Nutrition education and promotion: The role of FNS in helping low-income families make healthier eating and lifestyle choices [Internet]. FNS-USDA Food and Nutrition Service; 2010 [fecha de acceso: agosto de 2018]. Disponible en: https://fns-prod.azureedge.net/sites/default/ files/NutritionEdRTC.pdf

13. Thompson FE, Subar AF. Dietary assessment methodology. En: Coulston AM, Boushey $C J$, Ferruzzi MG, editores. Nutrition in the prevention and treatment of diseases. $3^{\text {rd }}$ ed. San Diego: Academic Press; 2012.

14. European Food Safety Authority. General principles for the collection of national food consumption data in the view of a pan-European dietary survey. EFSA Journal. 2009;7:1435.

15. Gersovitz M, Madden JP, Smiciklas-Wright H. Validity of the 24-hr dietary recall and seven-day record for group comparisons. J Am Diet Assoc. 1978;73:48-55.

16. Ortega RM, Pérez-Rodrigo C, López-Sobaler AM. Dietary assessment methods: dietary records. Nutr Hosp. 2015;31(Suppl 3):S38-45.

17. García SM, González C, Rucci C, Ambrosino CV, Vidal JM, Fantuzzi GA, et al. Self-administered structured food record for measuring individual energy and nutrient intake in large cohorts: design and validation. Endocrinol Diabetes Nutr. 2018;65(9):524-32.

18. Gagliardino JJ, Etchegoyen G, Bourgeois M, Fantuzzi G, Garcia S, Gonzáles $\mathrm{G}$, et al. Prevención primaria de Diabetes tipo 2 en Argentina: estudio piloto en la Provincia de Buenos Aires. Rev Argent Endocrinol Metab. 2016;53(4):135-41.

19. ENNyS. Encuesta Nacional de Nutrición y Salud. Alimentos consumidos en Argentina. Resultados de la Encuesta Nacional de nutricion y Salud - ENNyS 2005 [Internet]. Buenos Aires: Ministerio de Salud de la Nación, 2012 [fecha de acceso: agosto de 2018]. Disponible en: www.msal.gob.ar/images/stories/bes/graficos/0000000259cnt-a10alimentos-consumidos-en-argentina.pdf

20. Guías Alimentarias para la Población Argentina, Buenos Aires 2016 [Internet]. Buenos Aires: Ministerio de Salud de la Nación [fecha de publicación: 2016]. Disponible en: http://www.msal.gob.ar/images/ stories/bes/graficos/0000001007cnt-2017-06_guia-alimentariapoblacion-argentina.pdf

21. Asociación Argentina de Dietistas y Nutricionistas Dietistas. Guías Alimentarias para la población argentina. Manual de Multiplicadores. Ministerio de salud de la Nación. Buenos Aires, Argentina; 2006.

22. El gasto de consumo de los hogares urbanos en la Argentina. Un análisis a partir de las mediciones de 1996/1997, 2004/2005 y 2012/2013 [Internet]. Ciudad Autónoma de Buenos Aires: Instituto Nacional de Estadística y Censos - INDEC, 2014 [fecha de publicación: 2014]. Disponible en: https://www.indec.gov.ar/ftp/cuadros/sociedad/engho_ serie47.pdf

23. Canasta básica alimentaria y canasta básica total. Historia, forma de cálculo e interpretación [Internet]. Argentina: Instituto Nacional de Estadística y Censos; 2012. [fecha de publicación: 2012]. Disponible 
en: https://www.indec.gov.ar/ftp/cuadros/sociedad/informe_canastas_ basicas.pdf

24. Sistema de Análisis y Registro de Alimentos. Consulta de composición química de los alimentos [Internet]. Argentina: Ministerio de Salud de la Nación, Dirección Nacional de Salud Materno Infantil. Disponible en: http://datos.dinami.gov.ar/sara/

25. Mazzei ME, Puchulu R, Rochaix MA. Tabla de composición química de alimentos CENEXA. Centro de Endocrinología Experimental y Aplicada (UNLP-CONICET). 2. ${ }^{\text {a }}$ ed. Argentina; 1995.

26. Closa SS, de Landeta MC. Tablas de composición de alimentos. Base de datos ARGENFOODS [Internet]. Buenos Aires, Argentina: Universidad Nacional de Luján; 2002. Disponible en: http://www.argenfoods. unlu.edu.ar/

27. Poslusna K, Ruprich J, de Vries JH, Jakubilova M, van't Veer P. Misreporting of energy and micronutrient intake estimated by food records and 24 hour recalls, control and adjustment methods in practice. $\mathrm{Br}$ J Nutr. 2009;101(Suppl 2):S73-85.

28. Castro-Quezada I, Ruano-Rodríguez C, Ribas-Barba L, Serra-Majem L. Misreporting in nutritional surveys: methodological implications. Nutr Hosp. 2015;31(Suppl 3):S119-27.

29. Trinidad Rodríguez I, Fernández Ballart J, Cucó Pastor G, Biarnés Jordà E , Arija Val V. Validación de un cuestionario de frecuencia de consumo alimentario corto: reproducibilidad y validez. Nutr Hosp. 2008;23:242-52.

30. Aranceta Bartrina J, Pérez Rodrigo C, Alberdi Aresti G, Varela Moreiras G, Serra-Majem L. Controversies about population, clinical or basic research studies related with food, nutrition, physical activity and lifestyle. Nutr Hosp. 2015;31(Suppl 3):S15-21.

31. Alvariñas J, Salzberg S. Embarazo y lactancia. En: de Girolami DH, González Infantino CA. Clínica y terapéutica en la nutrición del adulto.

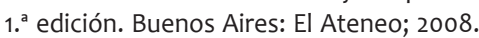

32. Committee on Nutritional Status During Pregnancy and Lactation. Nutrition during pregnancy: Part I. Weight gain: Part II. Washington: National Academies Press (US), Institute of Medicine; 1990.

33. McGowan CA, McAuliffe FM. Maternal nutrient intake and levels of energy underreporting. Eur J Clin Nutr. 2012;66:906-13.

34. Bourges H, Casanueva E, Rosado J, editores. Recomendaciones de ingestión de nutrimentos para la población Mexicana. Bases fisiológicas. Energía, proteínas, lípidos, hidratos de carbon y fibra. Tomo 2. México: Panamericana; 2008.

35. Recomendaciones nutricionales para la población cubana. Rev Cubana Invest Bioméd. 2009;28;9-16.

36. Jahns L, Conrad Z, Johnson LK, Scheett AJ, Stote KS, Raatz SK. Diet quality is lower and energy intake is higher on weekends compared with weekdays in midlife women: A 1-year cohort study. J Acad Nutr Diet. 2017;117:1080-6.

37. Thompson FE, Dixit-Joshi S, Potischman N, Dodd KW, Kirkpatrick SI, Kushi LH, et al. Comparison of interviewer-administered and automated self-administered 24-hour dietary recalls in 3 diverse integrated health systems. Am J Epidemiol. 2015;181:970-8.

38. Kirkpatrick SI, Subar AF, Douglass D, Zimmerman TP, Thompson FE, Kahle LL, et al. Performance of the Automated Self-Administered 24-hour Recall relative to a measure of true intakes and to an interviewer-administered 24-h recall. Am J Clin Nutr. 2014;100:233-40.

39. Cetin I, Laoreti A. The importance of maternal nutrition for health. Review. J Pediatr Neonat Individual Med. 2015;4(2):e040220.

40. Lee YQ, Collins CE, Gordon A, Rae KM, Pringle KG. The relationship between maternal nutrition during pregnancy and offspring kidney structure and function in humans: A systematic review. Nutrients. 2018;10:241.

41. Dodd JM, Whitehead CL. Obesity: Maternal obesity and congenital anomalies risk and diagnosis. Nat Rev Endocrinol. 2017;13:504-6. 